\title{
Existence of Generalized Homoclinic Solutions of Lotka-Volterra System under a Small Perturbation
}

\author{
Yuzhen Mi \\ Department of Mathematics, Lingnan Normal University, Zhanjiang, Guangdong 524048, China \\ Correspondence should be addressed to Yuzhen Mi; miyuzhen2009@126.com
}

Received 6 April 2016; Accepted 17 May 2016

Academic Editor: Zbigniew Leśniak

Copyright ( 2016 Yuzhen Mi. This is an open access article distributed under the Creative Commons Attribution License, which permits unrestricted use, distribution, and reproduction in any medium, provided the original work is properly cited.

\begin{abstract}
This paper investigates Lotka-Volterra system under a small perturbation $v_{x x}=-\mu\left(1-a_{2} u-v\right) v+\epsilon f\left(\epsilon, v, v_{x}, u, u_{x}\right), u_{x x}=-(1-u-$ $\left.a_{1} v\right) u+\epsilon g\left(\epsilon, v, v_{x}, u, u_{x}\right)$. By the Fourier series expansion technique method, the fixed point theorem, the perturbation theorem, and the reversibility, we prove that near $\mu=0$ the system has a generalized homoclinic solution exponentially approaching a periodic solution.
\end{abstract}

\section{Introduction}

The Lotka-Volterra system introduced by Lotka [1] and Volterra [2] is frequently used to describe the dynamics of biological systems and particularly plays an important role in studying population dynamics. Another reason for the importance of such system is that it has been successfully used in economic theory [3]. Many authors have studied the existence and uniqueness of stationary solution for a variety of Lotka-Volterra equations (e.g., Dancer [4] and Gui and Lou [5]). In recent years, it has been broadly studied in many aspects. Stability was discussed in Sakthivel et al. [6]. Travelling wave solutions were studied by Hosono [7] and Lin and Ruan [8]. The multiple periodic solutions were obtained in Wang et al. [9]. The bifurcation structures are given by Kan [10]. Limit cycles bifurcations were discussed by Wu and Liang [11]. The cyclicity of period annulus was analyzed in $\mathrm{Li}$ and Llibre [12].

In this paper, we will study the most well-known LotkaVolterra system

$$
\begin{aligned}
& v_{x x}=-\mu\left(1-a_{2} u-v\right) v, \\
& u_{x x}=-\left(1-u-a_{1} v\right) u,
\end{aligned}
$$

where $a_{1}, a_{2}$, and $\mu$ are constants and satisfy $\mu>0, a_{1}<1<$ $a_{2}$.
Motivated by [13, 14], we will study the existence of generalized homoclinic solutions of this Lotka-Volterra system. Since the real world always has some small noises or disturbances, here we are specially interested in the solutions of system (1) under small perturbations; that is, we will focus on the following system:

$$
\begin{aligned}
& v_{x x}=-\mu\left(1-a_{2} u-v\right) v+\epsilon f\left(\epsilon, v, v_{x}, u, u_{x}\right), \\
& u_{x x}=-\left(1-u-a_{1} v\right) u+\epsilon g\left(\epsilon, v, v_{x}, u, u_{x}\right),
\end{aligned}
$$

where $\epsilon$ is a small parameter and the general nonlinear terms $f$ and $g$ are smooth real functions. When $\epsilon=0$ this system has four equilibriums $(0,0,0,0),(0,0,1,0),(1,0,0,0)$, and $\left(\left(a_{2}-1\right) /\left(a_{1} a_{2}-1\right), 0,\left(a_{1}-1\right) /\left(a_{1} a_{2}-1\right), 0\right)$. When $\mu<0$ the linearized operator of (2) at $(1,0,0,0)^{T}$ has two pairs of purely imaginary eigenvalues. When $\mu=0$ the linearized operator of the system at $(1,0,0,0)^{T}$ has double real eigenvalues 0 and a pair of purely imaginary eigenvalues. When $\mu>0$ the linearized operator at $(1,0,0,0)^{T}$ has a positive eigenvalue, a negative eigenvalue, and a pair of purely imaginary eigenvalues. This yields that the linearized operator of system (2) has a saddle-center near $\mu=0$ while $\mu>0$, which means $\mu=0$ is the bifurcation point for system (2). We guess there might exist a homoclinic solution exponentially approaching a periodic solution (called generalized homoclinic solution) near $\mu=0$. Using the dynamical system approach, we will 
rigorously prove the existence of a generalized homoclinic solution (homoclinic solution exponentially tending to a small periodic solution at infinity) for $\mu>0$ but close to 0 , which corresponds to a generalized solitary wave solution of (2).

This paper is organized as follows. Section 2 derives the homoclinic solution of the dominant system of (2), which exponentially approaches zero at infinity. Section 3 shows that the system of (2) has a periodic solution. In Section 4, the fixed point theorem and the perturbation method yield that when higher order terms are added, the homoclinic solution of the dominant system deforms to a homoclinic solution exponentially approaching the periodic solution obtained in Section 3. This gives the existence of a generalized homoclinic solution of (2).

\section{Preliminary}

Let $v_{1}=v_{x}$ and $u_{1}=u_{x}$, and we change (2) into

$$
\begin{aligned}
v_{x} & =v_{1}, \\
v_{1 x} & =-\mu v+\mu v^{2}+a_{2} \mu u v+\epsilon f\left(\epsilon, v, v_{1}, u, u_{1}\right), \\
u_{x} & =u_{1}, \\
u_{1 x} & =-u+u^{2}+a_{1} u v+\epsilon g\left(\epsilon, v, v_{1}, u, u_{1}\right) .
\end{aligned}
$$

In this paper, we assume that $f$ and $g$ satisfy

$$
\begin{aligned}
f\left(\epsilon, 0,0, u, u_{1}\right) & =0, \\
f\left(\epsilon, v,-v_{1}, u,-u_{1}\right) & =f\left(\epsilon, v, v_{1}, u, u_{1}\right), \\
g(\epsilon, 0,0,0,0) & =0, \\
g\left(\epsilon, v,-v_{1}, u,-u_{1}\right) & =g\left(\epsilon, v, v_{1}, u, u_{1}\right) .
\end{aligned}
$$

Under assumption (4), system (3) is reversible with a reverser $S$ defined by

$$
S\left(v, v_{1}, u, u_{1}\right)=\left(v,-v_{1}, u,-u_{1}\right)
$$

that is, $S\left(v, v_{1}, u, u_{1}\right)(-x)$ is also a solution whenever $\left(v, v_{1}\right.$, $\left.u, u_{1}\right)(x)$ is a solution of (3). A solution $\left(v, v_{1}, u, u_{1}\right)(x)$ is reversible if $S\left(v, v_{1}, u, u_{1}\right)(-x)=\left(v, v_{1}, u, u_{1}\right)(x)$. This means that $v(x)$ and $u(x)$ are even functions and $v_{1}(x)$ and $u_{1}(x)$ are odd functions. The reversibility will play an important role in the existence of the generalized homoclinic solution.

Let

$$
\begin{aligned}
v & =w+1, \\
v_{1} & =w_{1}, \\
\widetilde{f}\left(\epsilon, w, w_{1}, u, u_{1}\right) & =f\left(\epsilon, w+1, w_{1}, u, u_{1}\right), \\
\widetilde{g}\left(\epsilon, w, w_{1}, u, u_{1}\right) & =g\left(\epsilon, w+1, w_{1}, u, u_{1}\right)
\end{aligned}
$$

and we have from (4)

$$
\begin{aligned}
& \tilde{f}\left(\epsilon, w,-w_{1}, u,-u_{1}\right)=\tilde{f}\left(\epsilon, w, w_{1}, u, u_{1}\right), \\
& \widetilde{g}\left(\epsilon, w,-w_{1}, u,-u_{1}\right)=\widetilde{g}\left(\epsilon, w, w_{1}, u, u_{1}\right) .
\end{aligned}
$$

Note that (6) changes system (3) into

$$
\begin{aligned}
w_{x} & =w_{1}, \\
w_{1 x} & =\mu w+\mu w^{2}+a_{2} \mu u(w+1)+\epsilon \tilde{f}\left(\epsilon, w, w_{1}, u, u_{1}\right), \\
u_{x} & =u_{1}, \\
u_{1 x} & =\left(a_{1}-1\right) u+u^{2}+a_{1} u w+\epsilon \tilde{g}\left(\epsilon, w, w_{1}, u, u_{1}\right) .
\end{aligned}
$$

System (8) can be written as

$$
\frac{d W}{d x}=L W+N_{1}(W)+N_{2}(W)+\epsilon R(\epsilon, W),
$$

where $W=\left(w, w_{1}, u, u_{1}\right)^{T}$ and

$$
L=\left(\begin{array}{cccc}
0 & 1 & 0 & 0 \\
\mu & 0 & 0 & 0 \\
0 & 0 & 0 & 1 \\
0 & 0 & a_{1}-1 & 0
\end{array}\right),
$$$$
N_{1}(W)=\left(\begin{array}{c}
0 \\
\mu w^{2} \\
0 \\
0
\end{array}\right) \text {, }
$$

$$
\begin{aligned}
& N_{2}(W)=\left(\begin{array}{c}
0 \\
a_{2} \mu u(w+1) \\
0 \\
u^{2}+a_{1} u w
\end{array}\right), \\
& R(\epsilon, W)=\left(\begin{array}{c}
\tilde{f}\left(\epsilon, w, w_{1}, u, u_{1}\right) \\
0 \\
\tilde{g}\left(\epsilon, w, w_{1}, u, u_{1}\right)
\end{array}\right) .
\end{aligned}
$$

From (7), system (9) is still reversible with the reverser $S$ if

$$
S\left(w, w_{1}, u, u_{1}\right)=\left(w,-w_{1}, u,-u_{1}\right) .
$$

We write the dominant system of (9) for small $u$ and $u_{1}$ as

$$
\frac{d W}{d x}=L W+N_{1}(W)
$$

which has a homoclinic solution $H(x)$ given by

$$
\begin{gathered}
H(x)=\left(-\frac{3}{2} \operatorname{sech}^{2}\left(\frac{\sqrt{\mu}}{2} x\right), \frac{3 \sqrt{\mu}}{2} \operatorname{sech}^{2}\left(\frac{\sqrt{\mu}}{2} x\right)\right. \\
\left.\cdot \tanh \left(\frac{\sqrt{\mu}}{2} x\right), 0,0\right)^{T}
\end{gathered}
$$

approaching zero as $x \rightarrow \pm \infty$. Moreover

$$
\begin{aligned}
S H(-x) & =H(x), \\
H(0) & =\left(-\frac{3}{2}, 0,0,0\right)^{T}
\end{aligned}
$$


and $H(x)$ satisfies the following inequality:

$$
|H(x)| \leq M e^{-\sqrt{\mu}|x|} \text { for any } x \in(-\infty, \infty),
$$

where $M$ is a positive constant.

In Section 4, we will prove the deformation of the homoclinic solution $H(x)$ for whole system (9). This demonstrates that system (3) at $(1,0,0,0)^{T}$ has a generalized homoclinic solution.

\section{Periodic Solutions}

By using the Fourier series expansion technique and the fixed point theorem, we will prove that (9) has a periodic solution which determines the forms of the generalized homoclinic solutions at infinity. Let

$$
\begin{aligned}
& C=\sqrt{1-a_{1}} u-i u_{1}, \\
& \tau=\sqrt{1-a_{1}}\left(1+r_{1}\right) x,
\end{aligned}
$$

where $r_{1}$ is a small real constant to be determined later. Then system (8) is changed into

$$
\begin{aligned}
w_{\tau} & =\frac{1}{\sqrt{1-a_{1}}\left(1+r_{1}\right)} w_{1}, \\
w_{1 \tau} & =\frac{\mu}{\sqrt{1-a_{1}}\left(1+r_{1}\right)} w+h_{1}\left(\mu, \epsilon, w, w_{1}, C, \bar{C}\right), \\
C_{\tau} & =\frac{i}{1+r_{1}} C+h_{2}\left(\mu, \epsilon, w, w_{1}, C, \bar{C}\right), \\
\bar{C}_{\tau} & =-\frac{i}{1+r_{1}} \bar{C}-h_{2}\left(\mu, \epsilon, w, w_{1}, C, \bar{C}\right),
\end{aligned}
$$

where $h_{1}$ is a real function and $h_{2}$ is a purely imaginary function and

$$
\begin{aligned}
& h_{1}\left(\mu, \epsilon, w, w_{1}, C, \bar{C}\right)=\frac{1}{\sqrt{1-a_{1}}\left(1+r_{1}\right)}\left[\mu w^{2}\right. \\
& \quad+\frac{a_{2} \mu}{2 \sqrt{1-a_{1}}}(C+\bar{C})(w+1) \\
& \left.\quad+\epsilon \tilde{f}\left(\epsilon, w, w_{1}, \frac{C+\bar{C}}{2 \sqrt{1-a_{1}}}, \frac{(C-\bar{C}) i}{2}\right)\right], \\
& h_{2}\left(\mu, \epsilon, w, w_{1}, C, \bar{C}\right) \\
& \quad=-\frac{i}{\sqrt{1-a_{1}}\left(1+r_{1}\right)}\left[\frac{(C+\bar{C})^{2}}{4\left(1-a_{1}\right)}+\frac{a_{1}(C+\bar{C}) w}{2 \sqrt{1-a_{1}}}\right. \\
& \left.\quad+\epsilon \tilde{g}\left(\epsilon, w, w_{1}, \frac{C+\bar{C}}{2 \sqrt{1-a_{1}}}, \frac{(C-\bar{C}) i}{2}\right)\right] .
\end{aligned}
$$

From (7) and (11), we may define

$$
S\left(w, w_{1}, C, \bar{C}\right)=\left(w,-w_{1}, \bar{C}, C\right),
$$

such that system (17) is reversible where we avoid again the introduction of a new notation.

Assume that

$$
\begin{aligned}
w(\tau) & =\sum_{n} w_{n} e^{i n \tau}, \\
w_{1}(\tau) & =\sum_{n} w_{1, n} e^{i n \tau}, \\
C(\tau) & =\sum_{n} C_{n} e^{i n \tau}, \\
\bar{C}(\tau) & =\sum_{n} \bar{C}_{n} e^{-i n \tau} .
\end{aligned}
$$

Plugging (20) into (17) and making the coefficient of each term in the Fourier series equal yield

$$
\begin{gathered}
w_{n}=-\frac{\sqrt{1-a_{1}}\left(1+r_{1}\right)}{n^{2}\left(1-a_{1}\right)\left(1+r_{1}\right)^{2}+\mu}\left[h _ { 1 } \left(\mu, \epsilon, w, w_{1}, C,\right.\right. \\
\bar{C})]_{n}, \\
w_{1, n}=-\frac{i\left(1-a_{1}\right)\left(1+r_{1}\right)^{2} n}{n^{2}\left(1-a_{1}\right)\left(1+r_{1}\right)^{2}+\mu}\left[h _ { 1 } \left(\mu, \epsilon, w, w_{1}, C,\right.\right. \\
\bar{C})]_{n}, \\
C_{n}=-\frac{i\left(1+r_{1}\right)}{n\left(1+r_{1}\right)-1}\left[h_{2}\left(\mu, \epsilon, w, w_{1}, C, \bar{C}\right)\right]_{n}, \\
\bar{C}_{n}=-\frac{i\left(1+r_{1}\right)}{n\left(1+r_{1}\right)-1}\left[h_{2}\left(\mu, \epsilon, w, w_{1}, C, \bar{C}\right)\right]_{-n},
\end{gathered}
$$

and for $n=1$

$$
\begin{aligned}
& r_{1} C_{1}=-i\left(1+r_{1}\right)\left[h_{2}\left(\mu, \epsilon, w, w_{1}, C, \bar{C}\right)\right]_{1}, \\
& r_{1} \bar{C}_{1}=-i\left(1+r_{1}\right)\left[h_{2}\left(\mu, \epsilon, w, w_{1}, C, \bar{C}\right)\right]_{-1},
\end{aligned}
$$

where $[h]_{k}$ denotes the $k$ th Fourier coefficient of $h$.

Now we activate $C_{1}$ and assume $\left|C_{1}\right|=I$. We first solve (21) for $w_{n}, w_{1, n}, C_{n}$, and $\bar{C}_{n}(n \neq 1)$ and then solve (22) for $r_{1}$.

Let $H^{m}(0,2 \pi)$ be a space of periodic functions of $\tau$ with a period $2 \pi$ such that their derivatives up to order $m$ are in $L^{m}(0,2 \pi)$; the norm is denoted by $\|\cdot\|_{m}$. Fix $C_{1}$ and define two spaces

$$
\begin{aligned}
H_{1}^{1} & (0.2 \pi) \\
& =\left\{f(\tau)=\sum_{n} f_{n} e^{i n \tau} \in H^{1}(0,2 \pi) \mid f_{1}=0\right\}, \\
H_{-1}^{1} & (0.2 \pi) \\
& =\left\{f(\tau)=\sum_{n} f_{n} e^{i n \tau} \in H^{1}(0,2 \pi) \mid f_{-1}=0\right\} .
\end{aligned}
$$


For $A, B \in H^{1}(0,2 \pi) \times H^{1}(0,2 \pi)$ and $D \in H_{1}^{1}(0,2 \pi)$, use (21) and we define a mapping $\Theta(A, B, D, \bar{D}, \alpha)$ from
$H^{1}(0,2 \pi) \times H^{1}(0,2 \pi) \times H_{1}^{1}(0,2 \pi) \times H_{-1}^{1}(0,2 \pi)$ to itself by

$$
\Theta(A, B, D, \bar{D}, \alpha)=\left(\begin{array}{c}
\sum_{n} \frac{-\sqrt{1-a_{1}}\left(1+r_{1}\right)}{n^{2}\left(1-a_{1}\right)\left(1+r_{1}\right)^{2}+\mu}\left[h_{1}\left(\mu, \epsilon, w, w_{1}, C, \bar{C}\right)\right]_{n} e^{i n \tau} \\
\sum_{n} \frac{-i n\left(1-a_{1}\right)\left(1+r_{1}\right)^{2}}{n^{2}\left(1-a_{1}\right)\left(1+r_{1}\right)^{2}+\mu}\left[h_{1}\left(\mu, \epsilon, w, w_{1}, C, \bar{C}\right)\right]_{n} e^{i n \tau} \\
\sum_{n \neq 1} \frac{-i\left(1+r_{1}\right)}{n\left(1+r_{1}\right)-1}\left[h_{2}\left(\mu, \epsilon, w, w_{1}, C, \bar{C}\right)\right]_{n} e^{i n \tau} \\
\sum_{n \neq 1} \frac{-i\left(1+r_{1}\right)}{n\left(1+r_{1}\right)-1}\left[h_{2}\left(\mu, \epsilon, w, w_{1}, C, \bar{C}\right)\right]_{-n} e^{-i n \tau}
\end{array}\right),
$$

where $\alpha=\left(\mu, \epsilon, r_{1}, C_{1}, \bar{C}_{1}\right)$. Assume that $B_{r}(0)$ is a ball with a radius $r$ in the space $H^{1}(0,2 \pi) \times H^{1}(0,2 \pi) \times H_{1}^{1}(0,2 \pi) \times$ $H_{-1}^{1}(0,2 \pi)$; we have the following lemma.

Lemma 1. For $(A, B, D, \bar{D}),\left(A_{1}, B_{1}, D_{1}, \bar{D}_{1}\right),\left(A_{2}, B_{2}, D_{2}, \bar{D}_{2}\right)$ $\in \bar{B}_{r}(0)$ and any small bounded $\alpha$ and $r, \Theta$ is smooth in its arguments and satisfies

$$
\begin{aligned}
& \|\Theta(A, B, D, \bar{D} ; \alpha)\|_{1} \leq M\left(\mu+|\epsilon|+\|A\|_{1}^{2}+\|D\|_{1}^{2}\right. \\
& \left.\quad+\left|C_{1}\right|^{2}\right), \\
& \left\|\Theta\left(A_{1}, B_{1}, D_{1}, \bar{D}_{1} ; \alpha\right)-\Theta\left(A_{2}, B_{2}, D_{2}, \bar{D}_{2} ; \alpha\right)\right\|_{1} \\
& \quad \leq M\left(\mu+|\epsilon|+\left|C_{1}\right|+\left\|A_{1}\right\|_{1}+\left|A_{2}\right|_{1}+\left\|D_{1}\right\|_{1}\right. \\
& \left.\quad+\left\|D_{2}\right\|_{1}\right)\left(\left\|A_{1}-A_{2}\right\|_{1}+\left\|D_{1}-D_{2}\right\|_{1}\right. \\
& \left.\quad+\left\|B_{1}-B_{2}\right\|_{1}\right) .
\end{aligned}
$$

Take $r=\left|C_{1}\right|$ and

$$
\begin{aligned}
& \mu=\mu_{1} I^{\beta_{1}}, \\
& \epsilon=\epsilon_{1} I^{\beta_{2}}, \\
& \beta_{1}>1, \\
& \beta_{2}>1,
\end{aligned}
$$

where $\mu_{1}, \epsilon_{1}, \beta_{1}$, and $\beta_{2}$ are fixed constants; Lemma 1 yields that $\Theta$ is a contraction mapping on $\bar{B}_{r}(0)$ for small $I$. Thus, $\Theta$ has a unique fixed point which is a smooth function of $\alpha$; write this fixed point as

$$
\left(w_{p}^{0}, w_{1 p}^{0}, C_{p}^{0}, \bar{C}_{p}^{0}\right)\left(\mu, \epsilon, r_{1}, C_{1}, \bar{C}_{1}\right)(\tau)
$$

which satisfies

$$
\left\|w_{p}^{0}\right\|_{1}+\left\|w_{1 p}^{0}\right\|_{1}+\left\|C_{p}^{0}\right\|_{1}+\left\|\widehat{C}_{p}^{0}\right\|_{1} \leq M\left(\mu+|\epsilon|+I^{2}\right) .
$$

Using the same argument we can show that (27) is in $H^{m}(0,2 \pi)$ and satisfies (28) with $H^{m}(0,2 \pi)$-norm for any integer $m>0$. We use $\left(w_{p}^{0}, w_{1 p}^{0}, C_{p}^{0}, \bar{C}_{p}^{0}\right)(\tau)$ to denote

$$
\left(w_{p}^{0}(\tau), w_{1 p}^{0}(\tau), C_{p}^{0}(\tau)+C_{1} e^{i \tau}, \bar{C}_{p}^{0}(\tau)+\bar{C}_{1} e^{-i \tau}\right) .
$$

Now we solve (22) for $r_{1}$. Substitute (27) into (22) and obtain

$$
r_{1}=g_{1}\left(\mu, \epsilon, r_{1}, C_{1}, \bar{C}_{1}\right),
$$

where

$$
\begin{aligned}
g_{1} & \left(\mu, \epsilon, r_{1}, C_{1}, \bar{C}_{1}\right) \\
& =\frac{i\left(1+r_{1}\right)\left[h_{2}\left(\mu, \epsilon, w, w_{1}, C, \bar{C}\right)\right]_{1}}{C_{1}}
\end{aligned}
$$

is smooth when $\mu, \epsilon, r_{1}, C_{1}, \bar{C}_{1}$ are near 0 . According to (26) and (18) we get $\left|g_{1}\right| \leq M(\mu+|\epsilon|+I)$, so $g_{1}$ is a contraction mapping. By the fixed point theorem, $g_{1}$ has a unique fixed point

$$
r_{1}=r_{1}(\mu, \epsilon, I)
$$

as a smooth real function for small $(\mu, \epsilon, I)$ satisfying

$$
\left|r_{1}\right| \leq M(\mu+|\epsilon|+I) .
$$

Therefore, (17) has a periodic solution

$$
\begin{gathered}
\left(w_{p}(\mu, \epsilon, I)(\tau), w_{1 p}(\mu, \epsilon, I)(\tau), C_{p}(\mu, \epsilon, I)\right. \\
\left.\cdot(\tau), \bar{C}_{p}(\mu, \epsilon, I)(\tau)\right)
\end{gathered}
$$

in $H^{m}(0,2 \pi)$ if $I \in\left(0, I_{1}\right]$ and (26) holds where $I_{1}$ is a fixed small positive constant.

By the relation $\tau=\sqrt{1-a_{1}}\left(1+r_{1}\right) x$, we write the periodic solution $\left(w_{p}, w_{1 p}, C_{p}, \bar{C}_{p}\right)(\tau)$ as $\left(w_{p}(\mu, \epsilon, I)(x), w_{1 p}(\mu\right.$, $\left.\epsilon, I)(x), C_{p}(\mu, \epsilon, I)(x), \bar{C}_{p}(\mu, \epsilon, I)(x)\right)$ with the frequency

$$
w_{1}(\mu, \epsilon, I)=\sqrt{1-a_{1}}\left(1+r_{1}(\mu, \epsilon, I)\right)
$$


for $I \in\left(0, I_{1}\right]$. Letting $C_{p}=\sqrt{1-a_{1}} u_{p}-i u_{1 p}$, we have

$$
\begin{gathered}
u_{p}(-x)=u_{p}(x), \\
u_{1 p}(-x)=-u_{1 p}(x) .
\end{gathered}
$$

Define

$$
\begin{aligned}
X_{\mu, \epsilon, I}(x) & =\left(w_{p}, w_{1 p}, u_{p}, u_{1 p}\right)^{T}(x) \\
& =\left(w_{p}, w_{1 p}, \frac{C_{p}+\bar{C}_{p}}{2 \sqrt{1-a_{1}}}, i \frac{C_{p}-\bar{C}_{p}}{2}\right)^{T}(x),
\end{aligned}
$$

which is smooth for $x$ and small $(\mu, \epsilon, I)$ with condition (26). Then, $X_{\mu, \epsilon, I}(x)$ is a reversible periodic solution of (9) under the reserve $S$ with frequency $w_{1}(\mu, \epsilon, I)$ which from (28) satisfies that for any integer $m>0$

$$
\left\|X_{\mu, \epsilon, I}(x)\right\|_{m} \leq M(\mu+|\epsilon|+I) .
$$

The Sobolev embedding theorem gives that (38) holds in $C_{B}^{m}(\mathbf{R})$-norm, which is a space of continuously differentiable functions up to the order $m$ with a supreme norm.

\section{Existence of Generalized Homoclinic Solutions}

In this section we demonstrate that (9) has a generalized homoclinic solution exponentially approaching the periodic solution $X_{\mu, \epsilon, I}$ obtained in Section 3.

Theorem 2. Suppose that assumption (4) holds. There exist constants $I_{0}>0, \mu_{1}>0$, and $\epsilon_{1}$ such that, for $I \in\left(0, I_{0}\right]$, if the small parameters $\mu=\mu_{1} I^{3 / 2}$ and $\epsilon=\epsilon_{1} I^{3 / 2}$, then (3) has a generalized homoclinic solution which is reversible and exponentially approaches the periodic solutions $(1,0,0,0)^{T}+$ $X_{\mu, \epsilon, I}(x+\theta)$ as $x \rightarrow \infty$ and $(1,0,0,0)^{T}+S X_{\mu, \epsilon, I}(-x+\theta)$ as $x \rightarrow-\infty$, where the phase shift $\theta$ is a continuous function in $I$ and the operator $S$ is defined in (5).

We divide the proof into two steps. Using the relationship between (3) and (9), we will first prove that (9) has a solution for $x \in[0, \infty)$, which exponentially approaches the periodic solution $X_{\mu, \epsilon, I}$ for some phase shift $\theta$ as $x \rightarrow \infty$. Then we solve for $\theta$ as a function of $\mu, \epsilon$, and $I$. This yields that this solution can be extended to $x \in(-\infty, 0]$ by using the reversibility.

Step 1 (existence of generalized homoclinic solution for $x \in[0, \infty))$. Assume that the solution $U(x)$ of (9) has the following form:

$$
U(x)=H(x)+Z(x)+\varsigma(x) X_{\mu, \epsilon, I}(x+\theta),
$$

where $H(x)$ and $X_{\mu, \epsilon, I}(x+\theta)$ are defined in (13) and (37), respectively, the phase shift $\theta \in S^{1}=[-\pi, \pi]$ is a constant, and the cut-off function $\varsigma(x)$ is in $C^{\infty}(\mathbf{R}, \mathbf{R})$ satisfying $0 \leq$ $\varsigma(x) \leq 1$ and

$$
\varsigma(x)= \begin{cases}1 & |x| \geq 2, \\ 0 & |x| \leq 1\end{cases}
$$

where $Z(x)$ is a perturbation term to be determined, which exponentially tends to 0 as $x \rightarrow \infty$ so that $U(x)$ is a solution of (9) that approaches the periodic solution $X_{\mu, \epsilon, I}(x+\theta)$ as $x \rightarrow \infty$. Plugging (39) into (9) yields

$$
\frac{d Z}{d x}=\mathscr{L}(x) Z+\widetilde{N}(x, z)+\epsilon \widetilde{R}(x, \epsilon, z),
$$

where

$$
\begin{aligned}
\mathscr{L}(x) & =L+d N_{1}[H(x)] \\
= & \left(\begin{array}{cccc}
0 & 1 & 0 & 0 \\
\mu-3 \mu \operatorname{sech}^{2}\left(\frac{\sqrt{\mu}}{2} x\right) & 0 & 0 & 0 \\
0 & 0 & 0 & 1 \\
0 & 0 & a_{1}-1 & 0
\end{array}\right), \\
\widetilde{N}(x, z) & N_{1}\left(H(x)+z(x)+\varsigma(x) X_{\mu, \epsilon, I}(x+\theta)\right) \\
& -N_{1}(H(x))-d N_{1}[H(x)] Z(x) \\
& +N_{2}\left(H(x)+z(x)+\varsigma(x) X_{\mu, \epsilon, I}(x+\theta)\right) \\
& -\varsigma(x) N_{2}\left(X_{\mu, \epsilon, I}(x+\theta)\right) \\
& -\varsigma(x) N_{1}\left(X_{\mu, \epsilon, I}(x+\theta)\right), \\
\widetilde{R}(x, & \epsilon, z) \\
= & R\left(\epsilon, H(x)+z(x)+\varsigma(x) X_{\mu, \epsilon, I}(x+\theta)\right) \\
& -\varsigma(x) R\left(\epsilon, X_{\mu, \epsilon, I}(x+\theta)\right) \\
& -\frac{1}{\epsilon} \varsigma^{\prime}(x) X_{\mu, \epsilon, I}(x+\theta),
\end{aligned}
$$

and $d$ means taking the Fréchet derivative.

By (15) and (38) we can obtain the following lemma.

Lemma 3. If $\mu, \epsilon$, and $I$ are small and $|Z|+\left|Z_{1}\right|+\left|Z_{2}\right| \leq M_{0}$ for some positive constant $M_{0}$, then $\widetilde{N}$ and $\widetilde{R}$ satisfy for $x \geq 0$

$$
\begin{aligned}
& |\widetilde{N}(x, Z)| \leq M\left[\left(e^{-\sqrt{\mu} x}+|Z|\right)(\mu+|\epsilon|+I)+|Z|^{2}\right], \\
& \left|\widetilde{N}\left(x, Z_{2}\right)-\widetilde{N}\left(x, Z_{1}\right)\right| \\
& \quad \leq M\left[\mu+|\epsilon|+I+\left|Z_{1}\right|+\left|Z_{2}\right|\right]\left|Z_{2}-Z_{1}\right|, \\
& |\widetilde{R}(x, \epsilon, Z)| \\
& \quad \leq M\left[e^{-\sqrt{\mu} x}+|Z|+\frac{1}{|\epsilon|}(\mu+|\epsilon|+I) e^{-\sqrt{\mu} x}\right] \\
& \left|\widetilde{R}\left(x, \epsilon, Z_{2}\right)-\widetilde{R}\left(x, \epsilon, Z_{1}\right)\right| \leq M\left|Z_{2}-Z_{1}\right| .
\end{aligned}
$$


Lemma 4. The solution of (39) that decays to zero at infinity can be found as

$$
\begin{aligned}
Z= & \mathscr{F}(Z) \\
\triangleq & \int_{0}^{x}\left\langle\widetilde{N}(t, Z)+\epsilon \widetilde{R}(t, \epsilon, Z), s_{1}^{*}(t)\right\rangle d t s_{1}(x) \\
& -\sum_{j=2}^{4} \int_{x}^{\infty}\left\langle\widetilde{N}(t, Z)+\epsilon \widetilde{R}(t, \epsilon, Z), s_{j}^{*}(t)\right\rangle d t s_{j}(x) .
\end{aligned}
$$

Proof.

$$
\frac{d Z(x)}{d x}=\mathscr{L}(x) Z(x)
$$

has four linearly independent solutions

$$
\begin{aligned}
& s_{1}(x)=\left\{\frac{3 \sqrt{\mu}}{2} \operatorname{sech}^{2}\left(\frac{\sqrt{\mu}}{2} x\right) \tanh \left(\frac{\sqrt{\mu}}{2} x\right),-\frac{3 \mu}{2}\right. \\
& \left.\cdot \operatorname{sech}^{2}\left(\frac{\sqrt{\mu}}{2} x\right)+\frac{3 \mu}{4} \operatorname{sech}^{4}\left(\frac{\sqrt{\mu}}{2} x\right), 0,0\right\}^{T} \text {, } \\
& s_{2}(x)=\left\{\frac { 4 } { 3 \mu } \operatorname { s e c h } ^ { 2 } ( \frac { \sqrt { \mu } } { 2 } x ) \operatorname { t a n h } ( \frac { \sqrt { \mu } } { 2 } x ) \left(\frac{15 \sqrt{\mu}}{16} x\right.\right. \\
& \left.-\operatorname{coth} \frac{\sqrt{\mu}}{2} x+\frac{1}{2} \sinh (\sqrt{\mu} x)+\frac{1}{32} \sinh (2 \sqrt{\mu} x)\right), \\
& -\frac{2 \sqrt{\mu}}{3 \mu}\left(2 \operatorname{sech}^{2}\left(\frac{\sqrt{\mu}}{2} x\right)-3 \operatorname{sech}^{4}\left(\frac{\sqrt{\mu}}{2} x\right)\right) \\
& \cdot\left(\frac{15 \sqrt{\mu}}{16} x-\operatorname{coth} \frac{\sqrt{\mu}}{2} x+\frac{1}{2} \sinh (\sqrt{\mu} x)\right. \\
& \left.+\frac{1}{32} \sinh (2 \sqrt{\mu} x)\right)+\frac{2 \sqrt{\mu}}{3 \mu} \operatorname{sech}^{2}\left(\frac{\sqrt{\mu}}{2} x\right) \\
& \cdot \tanh \left(\frac{\sqrt{\mu}}{2} x\right)\left(\frac{15}{8}+\operatorname{coth}^{2}\left(\frac{\sqrt{\mu}}{2} x\right)\right. \\
& \left.\left.+\cosh (\sqrt{\mu} x)+\frac{1}{8} \cosh (2 \sqrt{\mu} x)\right), 0,0\right\}^{T}, \\
& s_{3}(x)=\left\{0,0, \cos \left(\sqrt{1-a_{1}} x\right),-\sqrt{1-a_{1}}\right. \\
& \left.\cdot \sin \left(\sqrt{1-a_{1}} x\right)\right\}^{T}, \\
& s_{4}(x)=\left\{0,0, \sin \left(\sqrt{1-a_{1}} x\right), \sqrt{1-a_{1}}\right. \\
& \left.\cdot \cos \left(\sqrt{1-a_{1}} x\right)\right\}^{T},
\end{aligned}
$$

which satisfy

$$
\begin{aligned}
\left|s_{1}(x)\right| \leq M e^{-\sqrt{\mu} x}, \\
\left|s_{2}(x)\right| \leq M e^{\sqrt{\mu} x}, \\
\left|s_{3}(x)\right|+\left|s_{4}(x)\right| \leq M,
\end{aligned}
$$

for $x \in[0, \infty)$, and

$$
\begin{aligned}
& s_{1}(0)=\left\{0,-\frac{3 \mu}{4}, 0,0\right\}^{T}, \\
& s_{2}(0)=\left\{\frac{4}{3 \mu}, 0,0,0\right\}^{T}, \\
& s_{3}(0)=\{0,0,1,0\}^{T}, \\
& s_{4}(0)=\left\{0,0,0, \sqrt{1-a_{1}}\right\}^{T} .
\end{aligned}
$$

The adjoint equation of (45) has four linearly independent solutions given by

$$
\begin{aligned}
& s_{1}^{*}(x)=\left\{-\frac{2 \sqrt{\mu}}{3 \mu}\left(2 \operatorname{sech}^{2}\left(\frac{\sqrt{\mu}}{2} x\right)\right.\right. \\
& \left.-3 \operatorname{sech}^{4}\left(\frac{\sqrt{\mu}}{2} x\right)\right)\left(\frac{15 \sqrt{\mu}}{16} x-\operatorname{coth} \frac{\sqrt{\mu}}{2} x\right. \\
& \left.+\frac{1}{2} \sinh (\sqrt{\mu} x)+\frac{1}{32} \sinh (2 \sqrt{\mu} x)\right)+\frac{2 \sqrt{\mu}}{3 \mu} \\
& \cdot \operatorname{sech}^{2}\left(\frac{\sqrt{\mu}}{2} x\right) \tanh \left(\frac{\sqrt{\mu}}{2} x\right)\left(\frac{15}{8}\right. \\
& +\operatorname{coth}^{2}\left(\frac{\sqrt{\mu}}{2} x\right)+\cosh (\sqrt{\mu} x) \\
& \left.+\frac{1}{8} \cosh (2 \sqrt{\mu} x)\right),-\frac{4}{3 \mu} \operatorname{sech}^{2}\left(\frac{\sqrt{\mu}}{2} x\right) \\
& \cdot \tanh \left(\frac{\sqrt{\mu}}{2} x\right)\left(\frac{15 \sqrt{\mu}}{16} x-\operatorname{coth} \frac{\sqrt{\mu}}{2} x\right. \\
& \left.\left.+\frac{1}{2} \sinh (\sqrt{\mu} x)+\frac{1}{32} \sinh (2 \sqrt{\mu} x)\right), 0,0\right\}^{T}, \\
& s_{2}^{*}(x)=\left\{\frac{3 \mu}{2} \operatorname{sech}^{2}\left(\frac{\sqrt{\mu}}{2} x\right)-\frac{9 \mu}{4} \operatorname{sech}^{4}\left(\frac{\sqrt{\mu}}{2} x\right),\right. \\
& \left.-\frac{3 \sqrt{\mu}}{2} \operatorname{sech}^{2}\left(\frac{\sqrt{\mu}}{2} x\right) \tanh \left(\frac{\sqrt{\mu}}{2} x\right), 0,0\right\}^{T} \text {, } \\
& s_{3}^{*}(x)=\left\{0,0, \cos \left(\sqrt{1-a_{1}} x\right),-\frac{1}{\sqrt{1-a_{1}}}\right. \\
& \left.\cdot \sin \left(\sqrt{1-a_{1}} x\right)\right\}^{T} \\
& s_{4}^{*}(x)=\left\{0,0, \sin \left(\sqrt{1-a_{1}} x\right), \frac{1}{\sqrt{1-a_{1}}}\right. \\
& \left.\cdot \cos \left(\sqrt{1-a_{1}} x\right)\right\}^{T}
\end{aligned}
$$

which satisfy

$$
\begin{gathered}
\left|s_{1}^{*}(x)\right| \leq M e^{\sqrt{\mu} x}, \\
\left|s_{2}^{*}(x)\right| \leq M e^{-\sqrt{\mu} x}, \\
\left|s_{3}^{*}(x)\right|+\left|s_{4}^{*}(x)\right| \leq M
\end{gathered}
$$


for each $x \in[0, \infty)$; we have

$$
\begin{aligned}
& \left\langle s_{i}(x), s_{j}^{*}(x)\right\rangle=0, \quad i \neq j \\
& \left\langle s_{i}(x), s_{i}^{*}(x)\right\rangle=1 \quad i, j=1,2,3,4,
\end{aligned}
$$

where $\langle\cdot, \cdot\rangle$ denotes the Euclidean inner product on $\mathbf{R}^{4}$. From the above conditions we can get the expression of $Z$ in Lemma 4.

Fix $\nu \in(0, \sqrt{\mu})$ and consider (44) as a fixed point problem in a Banach space

$$
\begin{aligned}
E_{v} & =\left\{Z \in C\left([0, \infty) \times S^{1}\right) \mid \sup _{x \in[0, \infty)}\left\{|Z(x, \theta)| e^{\nu x}\right\}\right. \\
& <\infty\}
\end{aligned}
$$

with the norm

$$
\|Z\|_{\nu}=\sup \left\{|Z(x, \theta)| e^{\nu x} \mid x \in[0, \infty), \theta \in S^{1}\right\} .
$$

It is easy to obtain the following lemma using Lemma 3, (15), (28), and (38).

Lemma 5. The function $\mathscr{F}$ satisfies

$$
\begin{aligned}
& \|\mathscr{F}(Z)\|_{\nu} \leq M\left[\left(1+\|Z\|_{\nu}\right)(\mu+|\epsilon|+I)+\|Z\|_{\nu}^{2}\right], \\
& \left\|\mathscr{F}\left(Z_{1}\right)-F\left(Z_{2}\right)\right\|_{\nu} \\
& \quad \leq M\left[\mu+|\epsilon|+I+\left\|Z_{1}\right\|_{\nu}+\left\|Z_{2}\right\|_{\nu}\right]\left\|Z_{1}-Z_{2}\right\|_{\nu}
\end{aligned}
$$

for $Z, Z_{1}, Z_{2} \in E_{\gamma}$.

For any fixed constant $\rho \in(0,1)$, we let $r=M I^{\rho}$ and

$$
\begin{aligned}
& \mu=\mu_{1} I^{\beta_{1}}, \\
& \epsilon=\epsilon_{1} I^{\beta_{2}}, \\
& \beta_{i}=\rho+\widetilde{\beta}_{i}>1,
\end{aligned}
$$

where $\widetilde{\beta}_{i}$ are positive constants for $i=1,2$. Thus, (26) is satisfied, and we can show from Lemma 4 that $\mathscr{F}$ is a contraction on $\bar{B}_{r}(0) \subset E_{\gamma}$ for small $I$. Therefore, (44) has a unique solution $Z(x, \theta, \mu, \epsilon, I)$ satisfying

$$
|Z(x, \theta, \mu, \epsilon, I)| \leq M I^{\rho}, \quad x \in[0, \infty) .
$$

Using the same argument as that for (56) and an extension of a contraction principle [15], we can show that $Z$ is smooth in its argument. Thus the solution $Z$ of (41) exists if $x$ is in a finite interval and an initial condition is given, so that $U(x, \mu, \epsilon, \xi, I)$ defined in (39) exists for $x \geq \tilde{x}_{0}$ with any fixed $\tilde{x}_{0} \in(-\infty, \infty)$.

Step 2 (reversible generalized homoclinic solution for $x \in(-\infty, \infty))$. Using (5), (39), and the relationship between $v$ and $w$ in (6), we may define

$$
\widetilde{U}(x)= \begin{cases}U(x ; \theta, \mu, \epsilon, I)+U_{1} & \text { for } x \geq 0, \\ S(U(-x ; \mu, \epsilon, \xi, I))+S U_{1} & \text { for } x \leq 0,\end{cases}
$$

where $U_{1}=(1,0,0,0)^{T}$. In this step, we show that system (3) has a reversible generalized homoclinic solution for $x \in$ $(-\infty,+\infty)$. Depending on the reversibility of systems, this problem is equivalent to solving the following equation:

$$
(I-S) \widetilde{U}(0 ; \theta, \mu, \epsilon, I)=0
$$

for $\theta$. By (14), the definition of the cut-off function $\varsigma(x)$ in (40), and $W=\left(w, w_{1}, u, u_{1}\right)^{T}$, it is easy to check that (58) is equivalent to

$$
\begin{aligned}
& w_{1}(0)=0, \\
& u_{1}(0)=0 .
\end{aligned}
$$

Using (48) and (44) we know that (59) holds automatically. Thus we only need study (60) which can be transformed to

$$
\int_{0}^{\infty}\left\langle\widetilde{N}(t, z)+\epsilon \widetilde{R}(t, \epsilon, z), s_{4}^{*}(t)\right\rangle d t=0 .
$$

Lemma 6. Under the assumptions of Theorem 2, (61) can be transformed to

$$
\theta=I^{1 / 2} \varphi(\theta, \mu, \epsilon, I)
$$

where $\varphi$ is differentiable with respect to its arguments and $\varphi$ and its derivative with respect to $\theta$ are uniformly bounded for small bounded $\mu, \epsilon$, and $I$.

The proof of Lemma 6 is given in the appendix.

Using the fixed point theorem and Lemma 3, we can solve (62) for $\theta$ as a smooth function of $\mu, \epsilon, I$, so (60) is true. Uniqueness of the solution for an initial value problem implies that $\widetilde{U}$ is a generalized homoclinic solution of (3) and $\widetilde{S U}(-x)=\widetilde{U}(x)$, which exponentially approaches the periodic solution $(1,0,0,0)^{T}+X_{\mu, \epsilon, I}(x+\theta)$ as $x \rightarrow \infty$ and the periodic solution $(1,0,0,0)^{T}+S\left(X_{\mu, \epsilon, I}(-x+\theta)\right)$ as $x \rightarrow-\infty$. This completes the proof of Theorem 2 .

\section{Appendix}

In this section, we will give the proof for Lemma 6. Let

$$
\begin{aligned}
C_{p} & =\sqrt{1-a_{1}} u_{p}-i u_{1 p}, \\
\tau & =\sqrt{1-a_{1}}\left(1+r_{1}\right) x,
\end{aligned}
$$

where $r_{1}, u_{p}$, and $u_{1 p}$ are given in (32) and (37), respectively, which yields

$$
\begin{gathered}
u_{p}=\frac{C_{p}+\bar{C}_{p}}{2 \sqrt{1-a_{1}}}, \\
u_{1 p}=\frac{C_{p}-\bar{C}_{p}}{2} i .
\end{gathered}
$$


Thus, $\left(w_{p}, w_{1 p}, C_{p}, \bar{C}_{p}\right)$ is a $2 \pi$-periodic solution of the following system:

$$
\begin{aligned}
w_{p \tau}= & \frac{1}{\sqrt{1-a_{1}}\left(1+r_{1}\right)} w_{1 p}, \\
w_{1 p \tau}= & \frac{\eta}{\sqrt{1-a_{1}}\left(1+r_{1}\right)} w_{p} \\
& +h_{1}\left(\mu, \epsilon, w_{p}, w_{1 p}, C_{p}, \bar{C}_{p}\right), \\
C_{p \tau}= & \frac{i}{1+r_{1}} C_{p}+h_{2}\left(\mu, \epsilon, w_{p}, w_{1 p}, C_{p}, \bar{C}_{p}\right), \\
\bar{C}_{p \tau}= & -\frac{i}{1+r_{1}} \bar{C}_{p}-h_{2}\left(\mu, \epsilon, w_{p}, w_{1 p}, C_{p}, \bar{C}_{p}\right),
\end{aligned}
$$

where $h_{1}$ and $h_{2}$ are given in (18). We can express $C_{p}(\tau)$ as

$$
C_{p}(\tau)=e^{i\left(\tau /\left(1+r_{1}\right)\right)} C_{p}(0)+\varphi(\tau),
$$

where

$$
\varphi(\tau)=\int_{0}^{\tau} e^{i\left((\tau-s) /\left(1+r_{1}\right)\right)} h_{2}\left(\mu, \epsilon, w_{p}, w_{1 p}, C_{p}, \bar{C}_{p}\right) d s .
$$

We know that the coefficient of $e^{i \tau}$ is $C_{1}=I$. Thus,

$$
\begin{aligned}
I & =\frac{1}{2 \pi} \int_{0}^{2 \pi} C_{p}(s) e^{-i s} d s \\
& =\frac{1}{2 \pi} \int_{0}^{2 \pi} e^{-i s}\left(e^{i\left(s /\left(1+r_{1}\right)\right)} C_{p}(0)+\varphi(s)\right) d s \\
& =\left(1+\tilde{\varphi}\left(r_{1}\right)\right) C_{p}(0)+\frac{1}{2 \pi} \int_{0}^{2 \pi} e^{-i s} \varphi(s) d s,
\end{aligned}
$$

where $\widetilde{\varphi}\left(r_{1}\right)=\left(\left(1+r_{1}\right) / i 2 \pi r_{1}\right)\left(1-e^{-i\left(2 \pi r_{1} /\left(1+r_{1}\right)\right)}\right)-1=O\left(r_{1}\right)$ and $\widetilde{\varphi}(0)=0$, which yields

$$
C_{p}(0)=\frac{1}{1+\widetilde{\varphi}\left(r_{1}\right)}\left(I-\frac{1}{2 \pi} \int_{0}^{2 \pi} e^{-i s} \varphi(s) d s\right) .
$$

So,

$$
\begin{aligned}
C_{p}(\tau)= & \frac{e^{i\left(\tau /\left(1+r_{1}\right)\right)}}{1+\widetilde{\varphi}\left(r_{1}\right)}\left(I-\frac{1}{2 \pi} \int_{0}^{2 \pi} e^{-i s} \varphi(s) d s\right) \\
& +\varphi(\tau)
\end{aligned}
$$

or

$$
\begin{aligned}
C_{p}(x)= & \frac{e^{i \sqrt{1-a_{1}} x}}{1+\widetilde{\varphi}\left(r_{1}\right)}\left(I-\frac{1}{2 \pi} \int_{0}^{2 \pi} e^{-i s} \varphi(s) d s\right) \\
& +\varphi\left(\sqrt{1-a_{1}}\left(1+r_{1}\right) x\right) .
\end{aligned}
$$

From (33), (38), (56), and the expression of $h_{2}$ in (18), we can get $\varphi(x)=O\left(\mu+|\epsilon|+I^{2}\right)$, so that $C_{p}(x)=O(I)$ for small $I$. Then we obtain by (A.2)

$$
\begin{aligned}
u_{p}(x)= & \frac{1}{\sqrt{1-a_{1}}} \operatorname{Re} C_{p}(x) \\
= & \frac{1}{\sqrt{1-a_{1}}} \cos \left(\sqrt{1-a_{1}} x\right) I \\
& +U_{1}(x, \mu, \epsilon, I),
\end{aligned}
$$

$$
\begin{aligned}
u_{1 p}(x) & =-\operatorname{Im} C_{p}(x) \\
& =-\sin \left(\sqrt{1-a_{1}} x\right) I+U_{2}(x, \mu, \epsilon, I),
\end{aligned}
$$

where $U_{1}(x)=O\left(\mu+|\epsilon|+I^{2}\right)$ and $U_{2}(x)=O\left(\mu+|\epsilon|+I^{2}\right)$.

By (13), (37), (42), and $W=\left(w, w_{1}, u, u_{1}\right)^{T}$, we know that (61) is changed into

$$
\begin{aligned}
& 0=\int_{0}^{\infty} \frac{1}{\sqrt{1-a_{1}}}\left(\left(u(s)+\varsigma(s) u_{p}(s+\theta)\right)^{2}\right. \\
& +a_{1}\left(u(s)+\varsigma(s) u_{p}(s+\theta)\right)\left(-\frac{3}{2} \operatorname{sech}^{2}\left(\frac{\sqrt{\mu}}{2} s\right)\right. \\
& +w(s)+\varsigma(s) w_{p}(s+\theta)-\varsigma(s) \\
& \cdot\left(u_{p}^{2}(s+\theta)+a_{1} u_{p}(s+\theta) w_{p}(s+\theta)\right) \\
& \left.\cdot \cos \sqrt{1-a_{1}} s\right)-\varsigma^{\prime}(s) u_{p}(s+\theta) \sin \left(\sqrt{1-a_{1}} s\right) \\
& \left.-\frac{1}{\sqrt{1-a_{1}}} \varsigma^{\prime}(s) u_{1 p}(s+\theta)\right) \cos \left(\sqrt{1-a_{1}} s\right) \\
& -\frac{1}{\sqrt{1-a_{1}}}\left(\epsilon \tilde { g } \left(\epsilon,-\frac{3}{2} \operatorname{sech}^{2}\left(\frac{\sqrt{\mu}}{2} s\right)+w(s)\right.\right. \\
& +\varsigma(s) w_{p}(s+\theta), \frac{3 \sqrt{\mu}}{2} \operatorname{sech}^{2}\left(\frac{\sqrt{\mu}}{2} s\right) \\
& \cdot \tanh \left(\frac{\sqrt{\mu}}{2} s\right)+w_{1}(s)+\varsigma(s) w_{1 p}(s+\theta), u(s) \\
& \left.\left.+\varsigma(s) u_{p}(s+\theta)\right), u_{1}(s)+\varsigma(s) u_{1 p}(s+\theta)\right) \\
& -\varsigma(s) \\
& \cdot \widetilde{g}\left(\epsilon, w_{p}(s+\theta), w_{1 p}(s+\theta), u_{p}(s+\theta), u_{1 p}(s\right. \\
& +\theta)) \cos \sqrt{1-a_{1}} s d s \text {. }
\end{aligned}
$$

For computational simplicity, we take $\rho=\widetilde{\beta}_{k}=3 / 4$ for $k=1,2$ such that (55) and (56) are satisfied. Thus, (A.12) is changed into

$$
\begin{aligned}
0= & I \int_{0}^{\infty}-\frac{1}{\sqrt{1-a_{1}}} \varsigma^{\prime}(s)\left(\cos \sqrt{1-a_{1}}(s+\theta)\right) \\
& \cdot \sin \left(\sqrt{1-a_{1}} s\right)-\sin \left(\sqrt{1-a_{1}}(s+\theta)\right) \\
& \cdot \cos \left(\sqrt{1-a_{1}} s\right) d s+Q(\theta, \mu, \epsilon, I)=\frac{1}{\sqrt{1-a_{1}}} \\
& \cdot \sin \left(\sqrt{1-a_{1}} \theta\right) I+Q(\theta, \mu, \epsilon, I)
\end{aligned}
$$

where $Q(\theta, \mu, \epsilon, I)=O\left(I^{3 / 2}\right)$, which is equivalent to

$$
\theta=I^{1 / 2} \varphi(\mu, \epsilon, \xi, I),
$$


where $\varphi(\mu, \epsilon, \beta, I)=-\left(1 / \sqrt{\left(1-a_{1}\right) I}\right) \arcsin \left(\sqrt{1-a_{1}} Q(\theta, \mu\right.$, $\epsilon, I) / I$ ) is uniformly bounded for small $\mu, \epsilon$, and $I$. We can check that $\varphi(\theta, \mu, \epsilon, I)$ is differentiable with respect to its arguments, and $\varphi(\theta, \mu, \epsilon, I)$ and its derivatives with respect to $\theta$ are uniformly bounded for small bounded $(\mu, \epsilon, I)$. This completes the proof of Lemma 6.

\section{Competing Interests}

The author declares that there are no competing interests regarding the publication of this paper.

\section{Acknowledgments}

The author thanks Professor Shengfu Deng for his discussion. This research was supported by the National Natural Science Foundation of China (11371314), Guangdong Natural Science Foundation of China (S2013010015957), Science and Technology Planning Project of Zhanjiang (2016B01178, 2015B01011), High-Level Talent Project of Colleges and Universities in Guangdong Province (QBS201501), and Natural Science Research Training Program of Lingnan Normal University (YL1503).

\section{References}

[1] A. E. Lotka, Elements of Mathematical Biology, Dover, New York, NY, USA, 1956.

[2] V. Volterra, Leçons sur la Théorie Mathématique de la Lutte pour la Vie, Gauthier Villars, Paris, France, 1931.

[3] G. Nasritdinov and R. Dalimov, "Limit cycle, trophic function and the dynamics of intersectoral interaction," Current Research Journal of Economic Theory, vol. 2, pp. 235-273, 2010.

[4] E. N. Dancer, "On the existence and uniqueness of positive solutions for competing species models with diffusion," Transactions of the American Mathematical Society, vol. 326, no. 2, pp. 829-859, 1991.

[5] C. Gui and Y. Lou, "Uniqueness and nonuniqueness of coexistence states in the Lotka-Volterra competition model," Communications on Pure and Applied Mathematics, vol. 47, no. 12, pp. 1571-1594, 1994.

[6] K. Sakthivel, N. Baranibalan, J.-H. Kim, and K. Balachandran, "Stability of diffusion coefficients in an inverse problem for the Lotka-Volterra competition system," Acta Applicandae Mathematicae, vol. 111, no. 2, pp. 129-147, 2010.

[7] Y. Hosono, "Traveling waves for a diffusive Lotka-Volterra competition model I: singular perturbations," Discrete and Continuous Dynamical Systems, Series B, vol. 3, no. 1, pp. 79-95, 2003.

[8] G. Lin and S. Ruan, "Traveling wave solutions for delayed reaction-diffusion systems and applications to diffusive LotkaVolterra competition models with distributed delays," Journal of Dynamics and Differential Equations, vol. 26, no. 3, pp. 583-605, 2014.

[9] J. F. Wang, X. N. Zhou, and L. H. Huang, "Hopf bifurcation and multiple periodic solutions in Lotka-Volterra systems with symmetries," Nonlinear Analysis: Real World Applications, vol. 14, no. 3, pp. 1817-1828, 2013.

[10] Y. Kan, "Bifurcation structure of stationary solutions of a LotkaVolterra competition model with diffusion," SIAM Journal on Mathematical Analysis, vol. 29, no. 2, pp. 424-436, 1998.
[11] K. Wu and H. Liang, "Limit cycles bifurcating from a quadratic reversible Lotka-Volterra system with a center and three saddles," Chinese Annals of Mathematics B, vol. 35, no. 1, pp. 25-32, 2014.

[12] C. Li and J. Llibre, "The cyclicity of period annulus of a quadratic reversible Lotka-Volterra system," Nonlinearity, vol. 22, no. 12, pp. 2971-2979, 2009.

[13] S. Deng and B. Guo, "Generalized homoclinic solutions of a coupled Schrödinger system under a small perturbation," Journal of Dynamics and Differential Equations, vol. 24, no. 4, pp. 761-776, 2012.

[14] S. Deng and S. Sun, "Existence of three-dimensional generalized solitary waves with gravity and small surface tension," Physica D, vol. 238, pp. 1735-1751, 2009.

[15] W. Walter, Gewohnliche Differential Gleichungen, Springer, New York, NY, USA, 1992. 


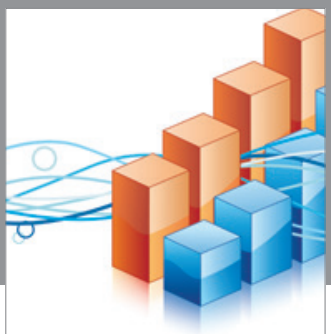

Advances in

Operations Research

vatem alat4

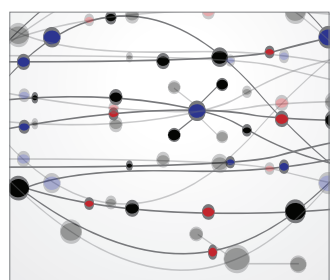

\section{The Scientific} World Journal
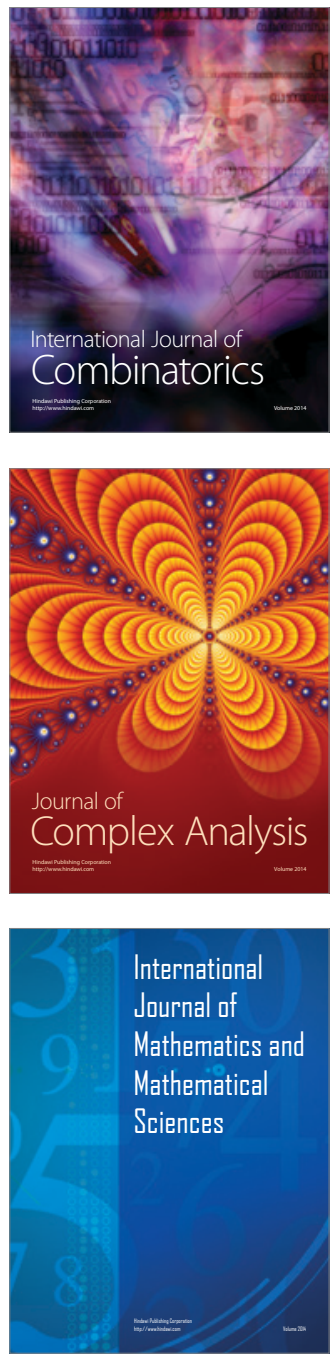
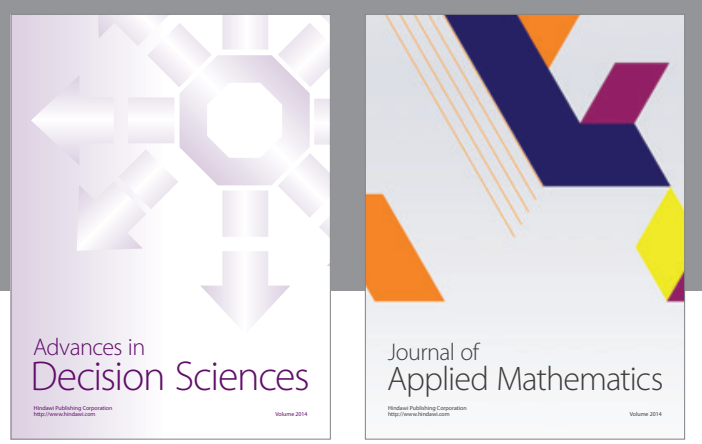

Algebra

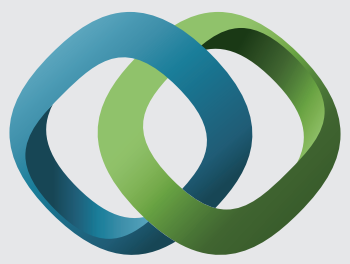

\section{Hindawi}

Submit your manuscripts at

http://www.hindawi.com
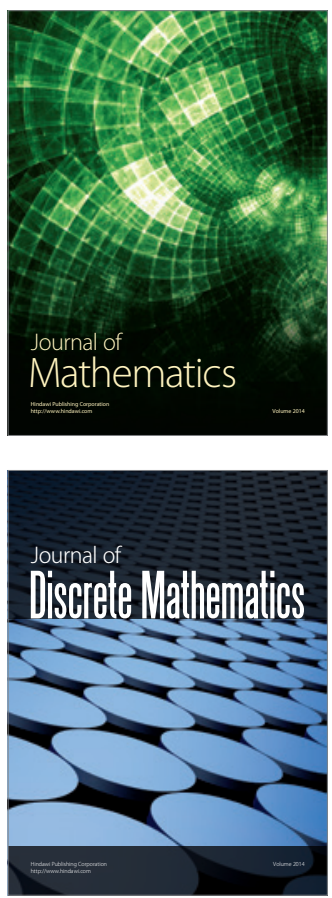

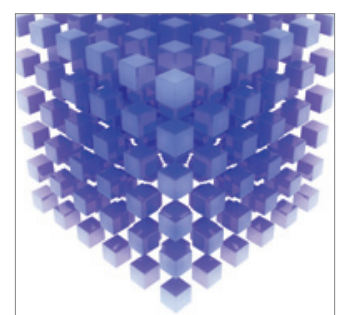

Mathematical Problems in Engineering
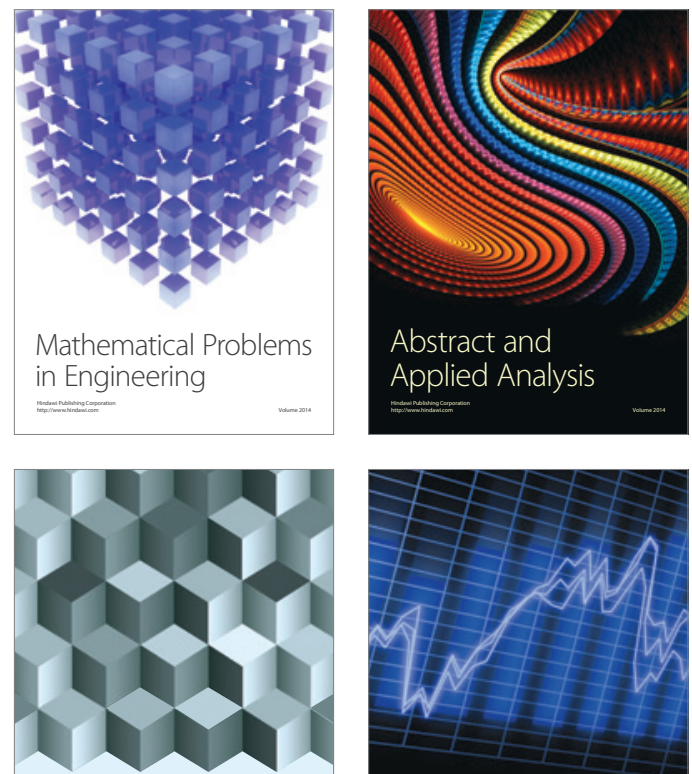

Journal of

Function Spaces

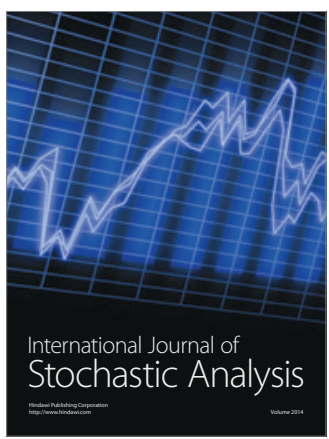

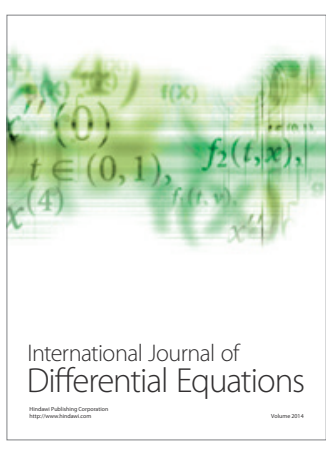
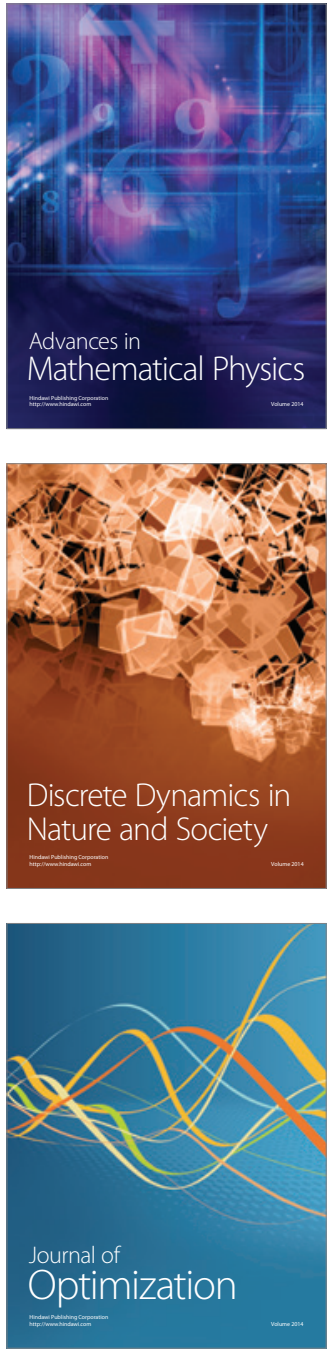